\title{
Vegetação Arbórea em Vertentes com Orientação Norte e Sul na Floresta Montana, Nova Friburgo-RJ
}

\author{
Erika Cortines ${ }^{1}$, André Luiz Pereira ${ }^{2}$, Pollyanna Rodrigues Oliveira dos Santos ${ }^{1}$, \\ Gilsonley Lopes Santos ${ }^{1}$, Ricardo Valcarcel ${ }^{1}$ \\ ${ }^{1}$ Departamento de Ciências Ambientais, Universidade Federal Rural do Rio de Janeiro - UFRRJ \\ ${ }^{2}$ Departamento de Botânica, Universidade Federal Rural do Rio de Janeiro - UFRRJ
}

\section{RESUMO}

Os diferentes atributos ambientais das vertentes norte e sul na Floresta Ombrófila Densa Montana da Serra do Mar podem explicar a variação da paisagem e a composição de espécies florestais dos seus ecossistemas. Foram amostradas 12 vertentes na zona do interflúvio de Nova Friburgo-RJ, sendo seis em cada face de exposição, utilizando o método fitossociológico Ponto por Quadrante. Os 480 indivíduos amostrados pertencem a 119 espécies, 75 gêneros e 40 famílias. A similaridade de espécies entre os dois tipos de vertentes foi de $36 \%$, em que 43 espécies foram comuns, 36 exclusivas da sul e 40 da norte. A espécie mais abundante foi Euterpe edulis L. , com 50 indivíduos na vertente sul e 29 na vertente norte. Conclui-se que a alta biodiversidade e a exposição das vertentes influenciam o padrão de similaridade/exclusividade das espécies.

Palavras-chave: diversidade, similaridade, Mata Atlântica.

\section{Arboreal Vegetation on the North and South Slopes of a Montana Forest in Nova Friburgo-RJ, Brazil}

\begin{abstract}
Different environmental attributes of north and south slopes of a Dense Ombrofilous Montana Forest in Serra do Mar can explain landscape variation and ecosystem forest species composition. Twelve slopes were assessed in the interfluve zone in Nova Friburgo, State of Rio de Janeiro, six on each slope exposition, using the point-centered quarter method on the north and south slopes. The 480 individuals evaluated belong to 119 species, 75 genera, and 40 families. The similarity between the slopes was $36 \%$, where 43 species were common to both north and south slopes, 36 species were exclusive to south slopes, and 40 were exclusive to north slopes. The most abundant species was Euterpe edulis L. with 50 individuals on south slopes and 29 on north slopes. It was concluded that high biodiversity and slopes exposition have an influence on species similarity/exclusivity patterns.
\end{abstract}

Keywords: diversity, similarity, Atlantic Forest. 


\section{INTRODUÇÃO}

A Floresta Atlântica abriga mais de 20 mil espécies vegetais, sendo um dos biomas reconhecidos como de máxima biodiversidade no planeta, além de apresentar o maior numero de espécies arbóreas entre os ecossistemas conhecidos (Pacto pela Restauração da Mata Atlântica, 2010). Uma das fisionomias características desse bioma é a Floresta Ombrófila Densa Montana (Velloso et al.,1991), caracterizada pela alta frequência de nuvens e baixo nível evapotranspirométrico (Hamilton et al., 1995), propiciando a formação de ecossistemas com serviços ambientais especiais em tempos de mudanças climáticas e suas consequências para os diversos segmentos socioambientais das bacias hidrográficas. Essas florestas ocupam altitudes que variam entre 500 e $1.500 \mathrm{~m}$, e estão localizadas em planaltos e encostas da Serra do Mar e da Mantiqueira (Ab'saber, 1992). Com a destruição das florestas tropicais, grande parte da sua biodiversidade se perde antes mesmo que seja conhecida (Borém \& Oliveira-Filho, 2002), inclusive na Mata Atlântica.

A região da Serra do Mar apresenta o maior remanescente de floresta contínua no Estado do Rio de Janeiro, englobando as seguintes Unidades de Conservação: Parque Estadual dos Três Picos (PETP), Área de Proteção Ambiental (APA) municipal dos Três Picos, APA da Caledônia, Reserva Ecológica de Guapiaçu, Parque Nacional da Serra dos Órgãos (PARNASO) e a Estação Ecológica do Paraíso, aumentando sua importância como corredor ecológico e refúgio para as espécies da fauna e da flora fluminense (INEA, 2009; SEABRA, 2009). A região apresenta grande significado hidrológico para a vertente atlântica, abastecendo parcialmente as cidades litorâneas e, em especial, as comunidades situadas na vertente direita da bacia do Rio Paraíba do Sul, onde se encontram cidades como Petrópolis, Teresópolis e Nova Friburgo, entre outras.

Nas regiões costeiras de serra, a orientação apresenta um papel determinante na conformação de regiões resilientes, onde a disponibilidade de atributos ambientais aumenta a riqueza e garante a presença (Marques et al., 2004) e a sustentabilidade dos fragmentos florestais. Na Serra do Mar, a disposição longitudinal das estruturas geológicas promove um efeito orográfico diferenciado na conformação das chuvas, na qual os índices pluviométricos apresentam-se superiores aos da região do entorno: superiores a $3.000 \mathrm{~mm}$ na vertente Atlântica (barlavento) e inferiores a $2.000 \mathrm{~mm}$ (sotavento) (Sant'anna Neto, 2005). Associados aos índices pluviométricos, há os efeitos orográficos das chuvas e as suas peculiaridades no tocante a reduzida frequência, baixa intensidade e duração, além da ocorrência microrregional. Nos ambientes a sotavento, é maior a ocorrência de pastagens, bem como a frequência de queimadas e desmatamentos, ao passo que as paisagens a barlavento são mais úmidas, biodiversas e propícias à presença de fragmentos florestais (Miranda \& Oliveira, 1983; Oliveira et al., 1995; Marcondes, 1996; Spolidoro, 1998; Marques et al., 2004). Neste estudo, a avaliação da vegetação foi feita nas vertentes norte e sul, a fim de se avaliar o efeito de perda/ganho de umidade e a adaptação das espécies arbóreas a essas condições diferenciadas.

O objetivo deste trabalho foi avaliar a diversidade da composição arbórea na face de exposição Sul (barlavento) e Norte (sotavento) em seis morrotes situados a partir do interflúvio $(5 \mathrm{~km})$ da Serra do Mar, no principal ponto de entrada das massas de ar úmidas vindas do Oceano Atlântico em direção ao Rio Paraíba do Sul, em Nova Friburgo-RJ.

\section{MATERIAL E MÉTODOS}

As unidades amostrais de vegetação arbórea estão localizadas entre as coordenadas UTM $22^{\circ} 21^{\prime} 21,52^{\prime \prime} \mathrm{S}$ e $42^{\circ} 39^{\prime} 58,58^{\prime \prime} \mathrm{O}$; $22^{\circ} 22^{\prime} 45,29^{\prime \prime} \mathrm{S}$ e $42^{\circ} 41^{\prime} 16,91^{\prime \prime}$ O, no município de Nova FriburgoRJ, na Fazenda Campestre, bairro de São Lourenço, próximo ao interflúvio da Serra do Mar, na cabeceira da Bacia do Rio Grande, afluente do Rio Paraíba do Sul (Figura 1).

O clima varia entre tropical de altitude (Cf) e subtropical (Cw) segundo Köppen, com precipitações médias anuais de $1.500 \mathrm{~mm}$; as temperaturas variam entre $9{ }^{\circ} \mathrm{C}$ (inverno) e $28^{\circ} \mathrm{C}$ (Verão), com média anual de $17,8{ }^{\circ} \mathrm{C}$. A umidade relativa é de $83 \%$ (Brasil, 1970).

Os principais solos são: Cambissolo Álico (Ca6), Litólico Álico (Ra), com presença de afloramentos 


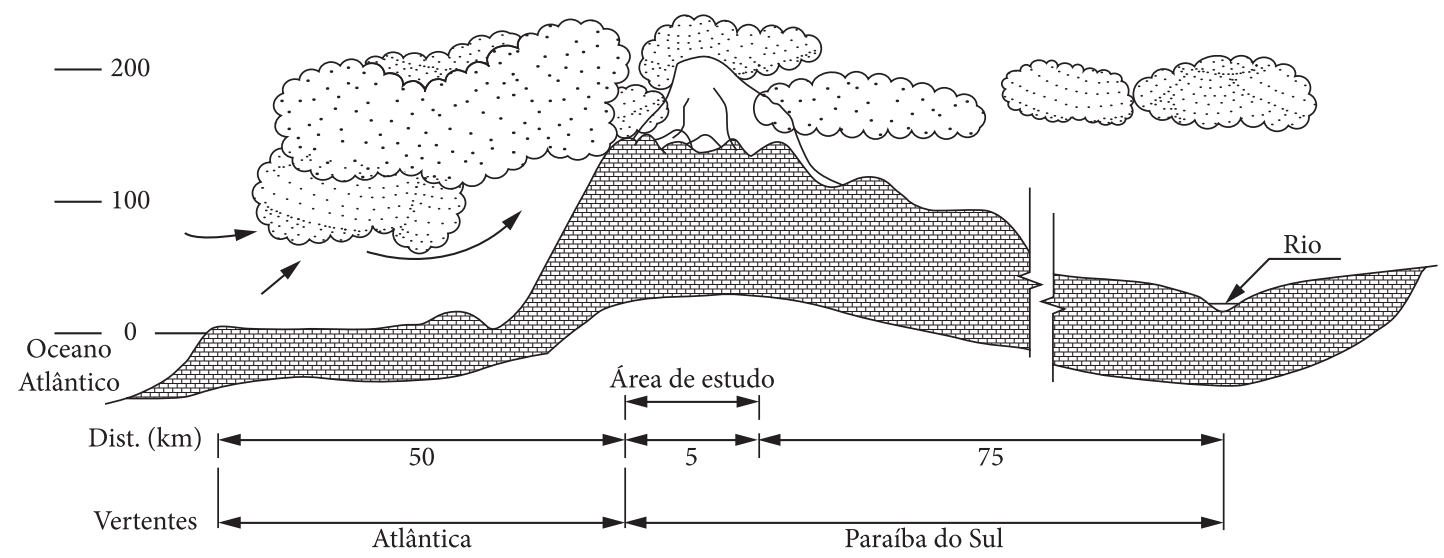

Figura 1. Corte longitudinal da Serra do Mar, com destaque para a área de estudo a sotavento da serra, na Bacia Hidrográfica do Rio Paraíba do Sul em Nova Friburgo-RJ.

Figure 1. Longitudinal section of the Serra do Mar, with emphasis on the study area downwind of the mountain, at the Paraiba do Sul watershed in Nova Friburgo-RJ, Brazil.

rochosos, e Neossolo. Nas várzeas, predominam os solos hidromórficos, amplamente utilizados para horticultura em razão da sua alta fertilidade e dos níveis de matéria orgânica (EMATER, 1994).

A topografia da bacia foi levantada a partir do Modelo Digital do Terreno utilizando-se software ArcGis 9.3 e curvas de níveis do IBGE, carta (Folha SF-23-Z-B-III-3) Nova Friburgo (Escala 1:50.000). A definição das unidades amostrais foi feita integrando-se os mapas de altitude, declividade e orientação das encostas. Os seis morros selecionados apresentam usos, declividade, altitude e orientação semelhantes, sendo o afastamento do interflúvio a principal diferença. A faixa de altitude na qual as linhas de amostragem ficaram contidas foi de 1050-1150 m.s.n.m. A declividade média das áreas de amostragem variou entre 20 e $40 \%$. Foram distribuídas 12 unidades amostrais (linhas) alocadas nas vertentes norte (sotavento) e sul (barlavento), em seis morros que se dispõem de forma sequencial nas áreas de várzea, a partir do divisor topográfico da Serra do Mar até uma distância de cerca de $5 \mathrm{~km}$ do mesmo (ver Figura 1).

Em cada linha, utilizou-se o método fitossociológico de Ponto por Quadrante (Cottan \& Curtis, 1956), ao longo de $50 \mathrm{~m}$ na encosta, com distância de $5 \mathrm{~m}$ entre cada ponto. As linhas foram todas alocadas no terço médio das vertentes, evitandose o contato com a várzea e os topos de morro. Em cada ponto, foram mensuradas quatro árvores com diâmetro à altura do peito (DAP) $>5 \mathrm{~cm}$, totalizando 40 indivíduos por linha. Foram mensuradas 480 árvores, sendo 240 em cada exposição. Os parâmetros mensurados em cada árvore foram: DAP a $1,30 \mathrm{~m}$ (Brokaw \& Thompson, 2000), diâmetro de copa (m) e altura da árvore $(\mathrm{m})$. Foram calculados os seguintes parâmetros: Frequência Absoluta (FA), Valor de Importância (VI), Índice de Shannon-Wiever ( $\mathrm{H}^{`}$ ) e Índice de Similaridade de Jaccard (J), descritos em Sylvestre \& Rosa (2002).

\section{RESULTADOS E DISCUSSÃO}

Foram amostradas 119 espécies, 75 gêneros e 40 famílias botânicas. Destas, 17 foram representadas por mais de uma espécie e 23 por apenas uma única espécie (Figura 2).

$\mathrm{Na}$ vertente norte, foram encontradas 85 espécies, enquanto que, na vertente sul, foram 84 espécies (Tabela 1)

As famílias que tiveram especificidade em relação à orientação das vertentes foram, na vertente norte: Meliaceae, Phylantaceae, Picramniaceae, Rosaceae, Styracaceae e Verbenaceae; na vertente sul: Anacardiaceae, Bignoniaceae, Fabaceae Caesalpinoideae, Chloranthaceae e Winteraceae.

$\mathrm{O}$ índice de diversidade de Shannon-Wiever foi de 4,0, quando consideradas todas as espécies (ambas as vertentes). Pereira et al. (2006), em 


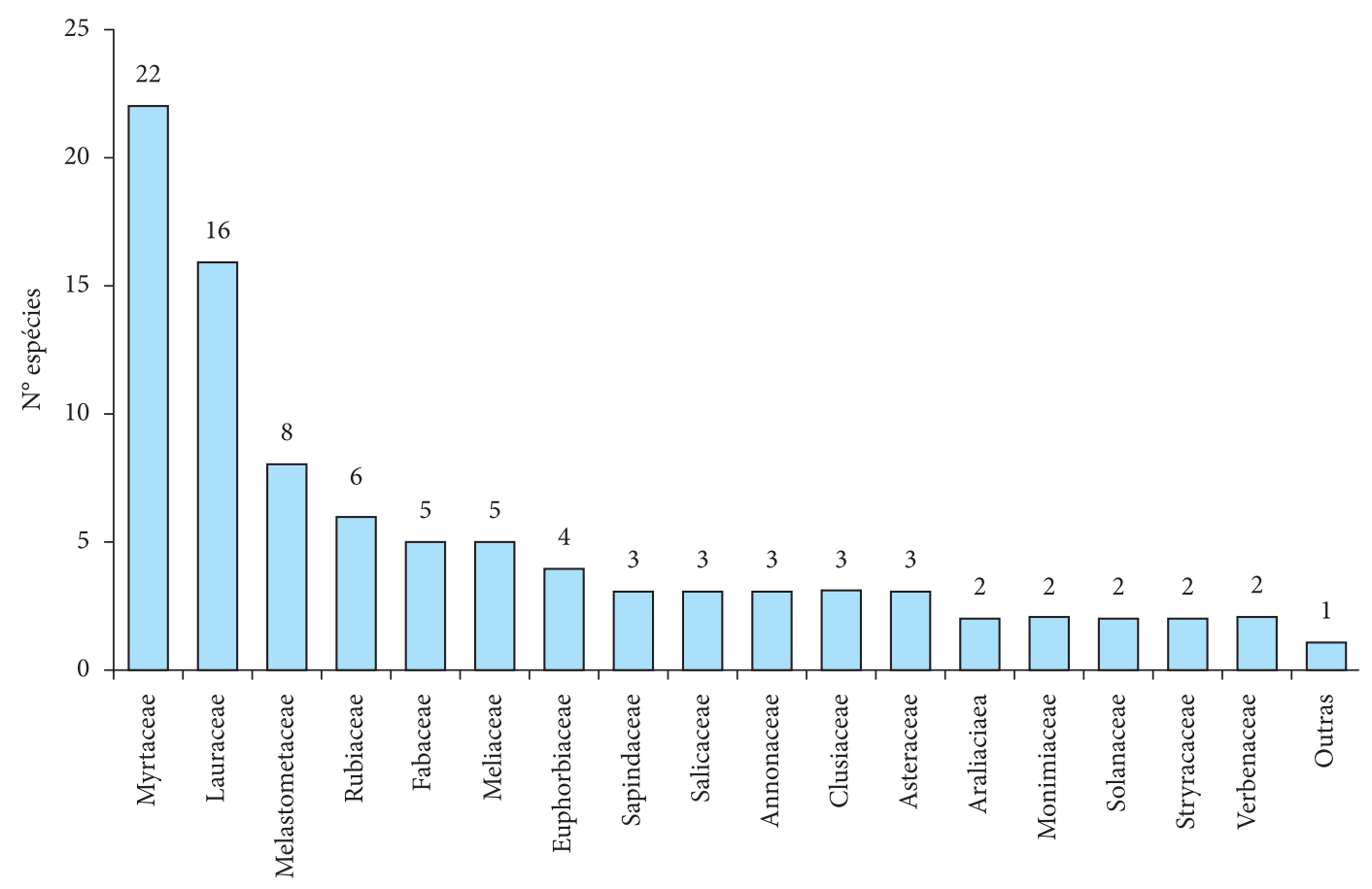

Figura 2. Famílias botânicas predominantes e respectiva riqueza de espécies em Floresta Montana de Nova Friburgo-RJ.

Figure 2. Predominant botanical families and it's respactive species richness at a Montana Forest of Nova Friburgo, RJ.

Tabela 1. Comparação dos índices ecológicos entre vertentes norte e sul, em uma Floresta-OmbrófilaDensa-Montana, entre as altitudes de 1050 e 1150 m, no município de Nova Friburgo-RJ.

Table 1. Comparison of ecological index between north and south slopes, on a Tropical Montana Forest, at an altitude of 1050-1150 m, county of Nova Friburgo, RJ.

\begin{tabular}{|c|c|c|}
\hline & SUL & NORTE \\
\hline Área amostrada $\left(\mathrm{m}^{2}\right)$ & 587 & 573 \\
\hline Índice de diversidade (H') & 3,8 & 3,9 \\
\hline $\begin{array}{l}\text { Índice de diversidade máximo } \\
\text { (H máx.) }\end{array}$ & 4,4 & 4,4 \\
\hline Riqueza de espécies & 85 & 84 \\
\hline Abundância de indivíduos & 240 & 240 \\
\hline Riqueza de famílias & 39 & 35 \\
\hline $\begin{array}{l}\text { Somatório área basal individual } \\
\left(\mathrm{m}^{2}\right)\end{array}$ & 3,2 & 1,3 \\
\hline Área basal por hectare $\left(\mathrm{m}^{2}\right)$ & 53,90 & 23,38 \\
\hline Espécies exclusivas & 33 & 35 \\
\hline Espécies em comum & 43 & 43 \\
\hline Famílias exclusivas & 5 & 6 \\
\hline
\end{tabular}

estudos comparativos entre vegetação arbórea de 20 fragmentos florestais do Sudeste do Brasil, encontraram maior diversidade em locais com heterogeneidade entre os aspectos topográficos e químicos do solo, e menor diversidade nas áreas mais próximas da borda. Barros (2006) comparou a diversidade entre áreas de borda e interior de fragmentos, na mesma bacia hidrográfica do presente estudo, em Nova Friburgo, e encontrou índice de diversidade de 3,42 para bordas agrícolas, de 3,85 em bordas de pastagem e de 3,37 no interior de fragmento. Para este estudo, a diversidade encontrada para a comparação entre as vertentes distintas demonstrou semelhança com 3,8 (sul) e 3,9 (norte), enaltecendo a importância dessas matas para a conservação da biodiversidade da Floresta Montana.

O índice de similaridade de espécies foi de $34 \%$ entre as vertentes, segundo o qual 43 espécies são comuns, 33 exclusivas da vertente sul e 35 exclusivas da vertente norte (Tabela 2); tal ocorrência pode ser uma evidência da adaptação das espécies às 


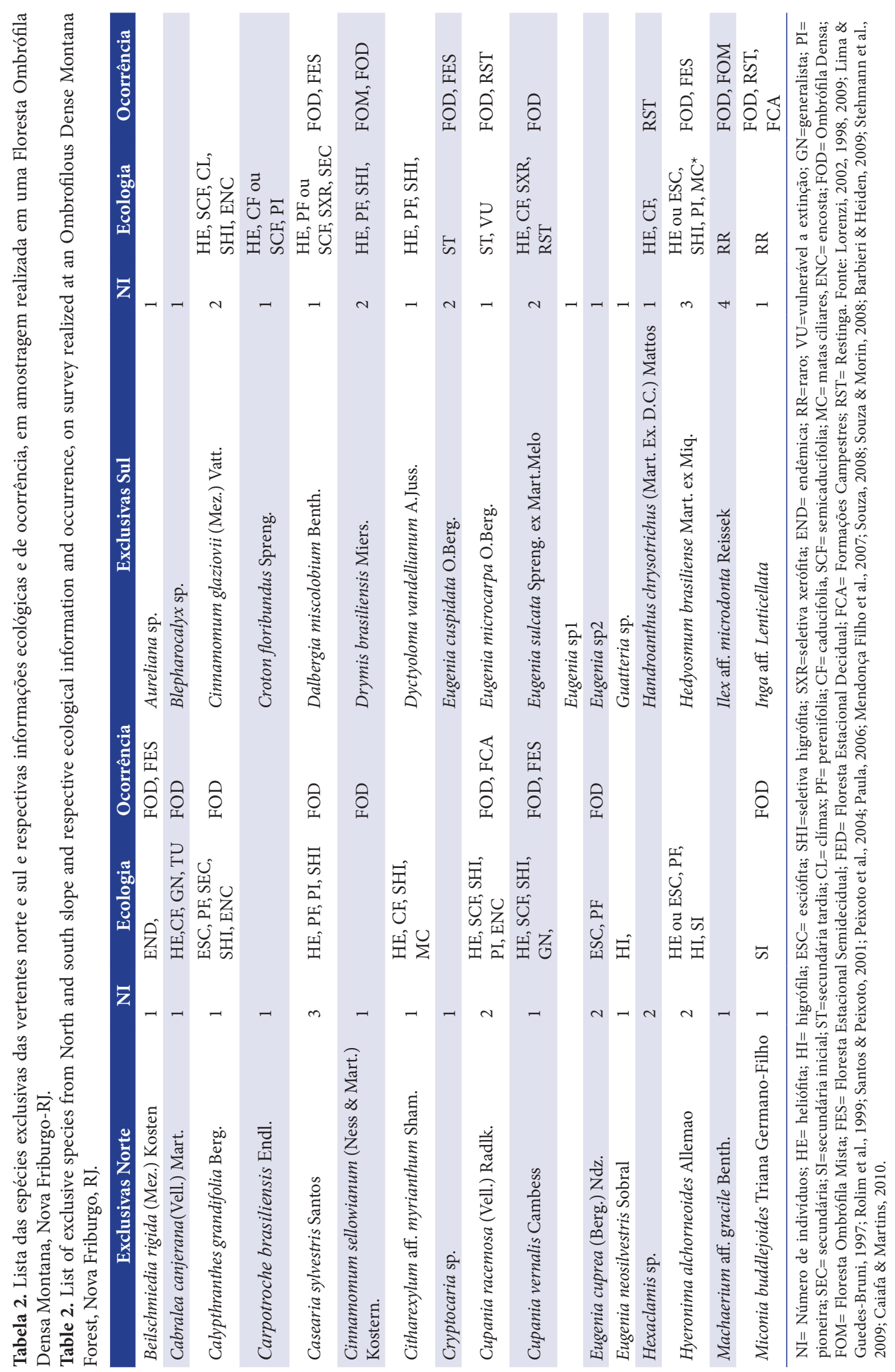




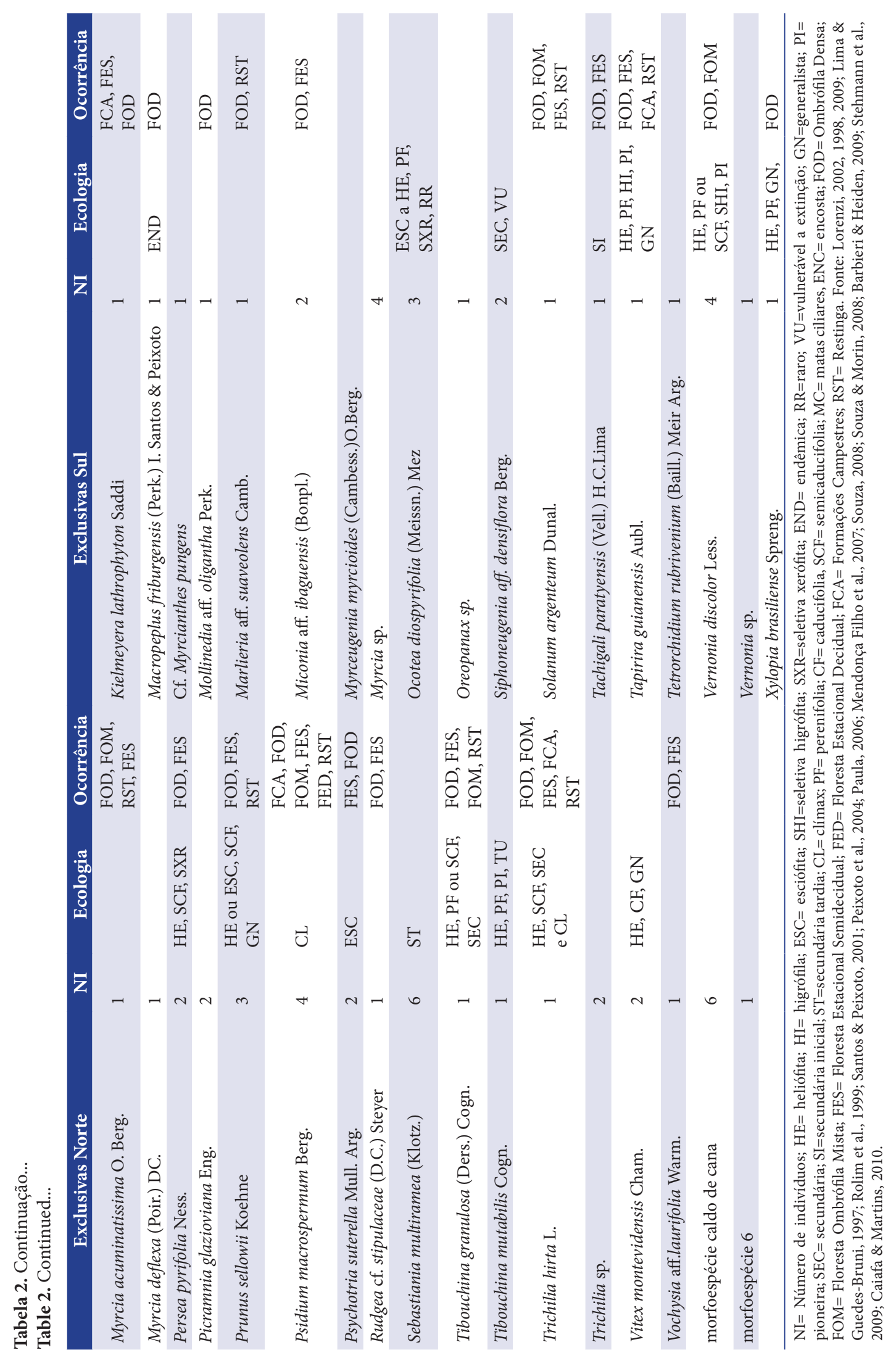


condições ambientais diferenciadas entre vertentes, corroborando a hipótese de que as plantas respondem às mudanças ambientais em escala local.

Na vertente norte, $30,8 \%$ das espécies exclusivas são também adaptadas aos ecossistemas de Floresta Estacional Semidecidual, enquanto que, na vertente sul, tal porcentagem foi de $22,2 \%$, sugerindo diferenças na umidade disponível para as plantas e levando, assim, a adaptação das espécies do norte à menor oferta de água. Uma provável justificativa para esse fato pode ser a intensidade de chuva oculta causada pela interceptação horizontal da umidade atmosférica entre as vertentes (barlavento e sotavento), principalmente nas épocas sem chuva. Essa adaptação às condições microclimáticas pode explicar a exclusividade de espécies encontradas entre as vertentes da região e o alto grau de endemismo desse bioma.

O papel dos nevoeiros, como fonte de água para a vegetação em áreas montanhosas, é conhecido, principalmente nas épocas sem chuva (Bruijnzeel, 2001). Nas florestas de Sequoia na Califórnia, Dawson (1998) observou que 13-45\% da água transpirada por essas árvores é proveniente da água vinda da interceptação de umidade atmosférica (nevoeiro).

Estudos relacionando topografia com a estrutura e a diversidade da floresta em Samoa encontraram diferenças estruturais entre as matas das cumeeiras de morro, de meia encosta e dos vales. As matas foram mais diversas nas áreas de topo, sendo, porém, mais baixas e densas; diversamente, as matas nos vales eram compostas por árvores maiores e em menor densidade e diversidade (Webb et al., 1999). Na África do Sul, análises sobre a dinâmica das espécies arbóreas e arbustivas demonstraram que duas variáveis, luz e água, são as principais responsáveis pelas variações na riqueza em nível de família, gênero e espécie (O`Brien, 2000).

As condições abióticas locais podem influenciar na composição de espécies. Estudos da vegetação arbustivo-arbórea realizados na Armação dos Búzios-RJ indicaram que a distribuição espacial das espécies parece estar relacionada às características ecológicas de cada encosta, como resultado de sua orientação, modificando a incidência de luminosidade, a recepção de ventos e a umidade, e condicionando composições e estruturas próprias à vegetação (Kurtz et al., 2009). Outro estudo na região de Cabo Frio-RJ encontrou maior diversidade de espécies nas vertentes com orientação Sul (Sá, 2006).

Nas unidades amostrais, o palmito-jussara (Euterpe edulis L. ) foi a espécie mais abundante em ambas as vertentes, totalizando 79 indivíduos amostrados (16,5\% do total), sendo 29 na vertente norte e 50 na vertente sul. Esses resultados concordam com estudos realizados na mesma região analisando efeito de borda, em que o E. edulis também foi a espécie mais representativa (Bohrer \& Barros, 2006). Essa espécie, apesar de estar contida na listagem de espécies ameaçadas de extinção (Brasil, 2008), apresentou grande importância ecológica nas linhas de amostragem. Uma análise das dez espécies mais abundantes (Figura 3) evidenciou que a maior frequência relativa foi alcançada por essa espécie (com 12 e 20,1\% para as vertentes norte e sul, respectivamente), seguida por Alchornea triplinervia (6,2; 5,8\%), Myrcia rufulla (5; 5\%) e Guapira opositta $(5 ; 3,8 \%)$.

$\mathrm{Na}$ vertente norte, $A$. triplinervia superou em VI o E. edulis, com os valores de 49,2 e 37,1 respectivamente. $\mathrm{Na}$ sequência, as espécies com maiores VI foram Guapira opposita (21,6), Myrcia ruffula (19,1), Gordonia fruticosa (15,1), cf. Daphnopsis $(14,4)$, Amaioua SP. $(14,2)$, Sebastiania multiramea $(13,6)$, Persea pyrifolia $(11,7)$, Vochysia aff. $(11,1)$, Clethra scabra $(10,9)$ e Eugenia neosilvestris $(10,8)$. Destas, S. multiramea, P. pyrifolia e E. neosilvestris foram exclusivas da vertente norte.

Na vertente sul, o VI das duas primeiras espécies referidas se inverteu: E. edulis obteve o maior VI, 50,9, contra 20,4 para a A. triplinervia. Em seguida, foram: M. ruffula $(13,2)$, Ocotea puberula $(10,8)$, G. opposita $(10,5)$, uma espécie não identificada $(9,6)$, Erythroxylum sp. (8,2), Psychotria sessilis $(6,8)$ e Cyathea sp. $(6,6)$. Todas as espécies de maior VI na vertente sul ocorreram também na norte. A espécie Cyathea sp., conhecida como samabaia-açu, ocorreu com apenas um indivíduo na vertente norte contra seis na vertente sul. Estudos com a espécie Cyathea planadae N.C. Arens \& A.R.Sm. indicaram que sua presença é restrita ao sub-bosque de florestas de copa adensada (Arens \& Sa'nchez Baracaldo, 1998; Arens \& Smith, 1998). Note-se que a espécie 


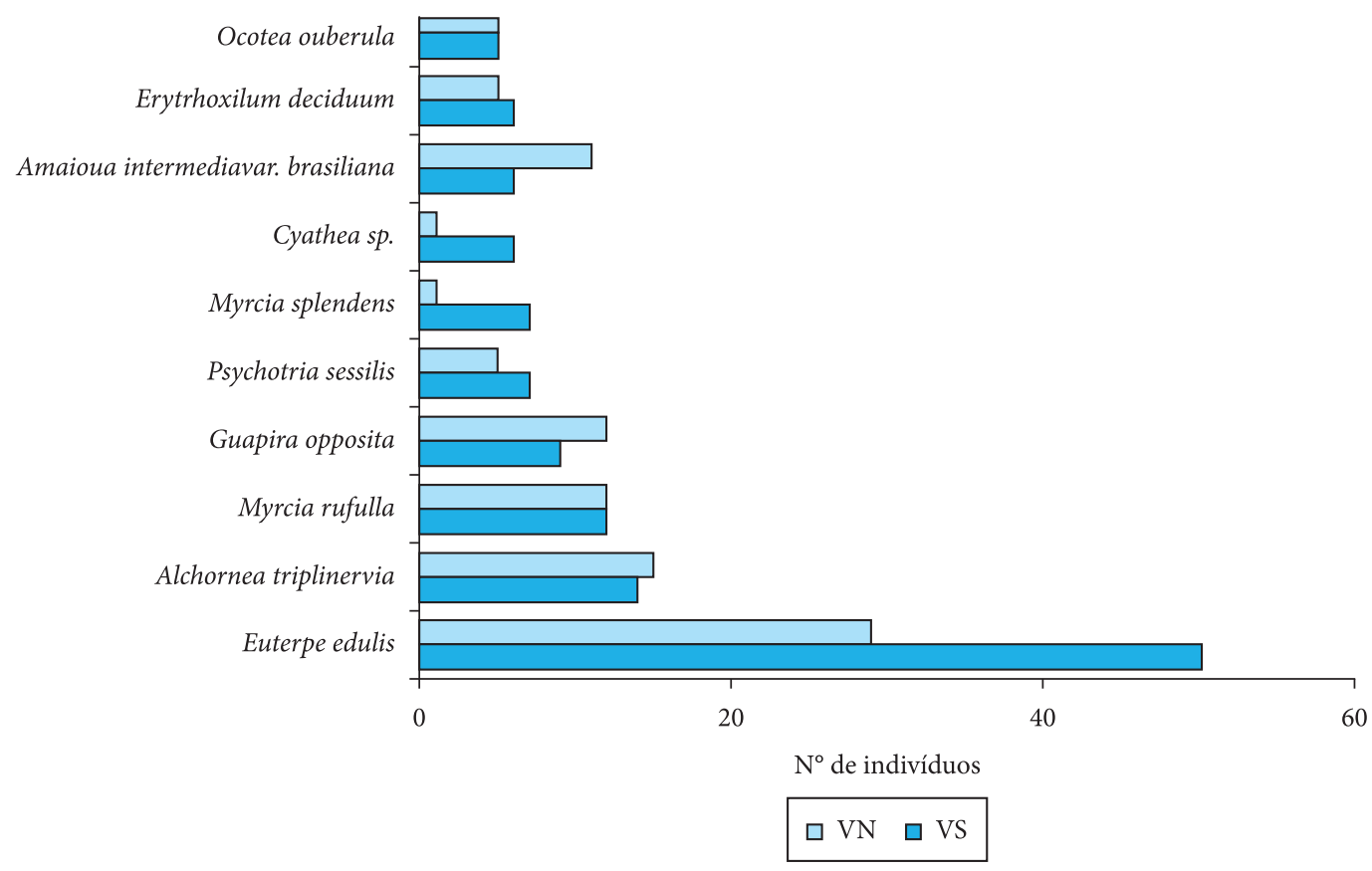

Figura 3. Abundância das 10 espécies de maior representatividade nas 12 linhas de amostragem da vegetação arbórea, em uma Floresta Ombrófila Densa Montana, no município de Nova Friburgo, RJ.

Figure 3. Abundance of the 10th most representative species on the 12 arboreal vegetation survey lines, at a Tropical Montana Forest, county of Nova Friburgo, RJ.

Cyathea caracasana (Klotzsch) foi considerada por Arens (2001) como generalista, persistindo nos ambientes sombreados ao longo da sucessão vegetal.

Os resultados encontrados neste estudo indicam que, se aprimorados os conhecimentos sobre as espécies exclusivas encontradas, poderão ser utilizadas essas adaptações das espécies para otimizar a eficiência de plantios em áreas de restauração ambiental similares às estudadas.

\section{CONCLUSÕES}

A orientação das vertentes influenciou a ocorrência de espécies arbóreas, cuja exclusividade encontrada pode estar relacionada com a alta biodiversidade das florestas amostradas. $\mathrm{Na}$ restauração ambiental, a priorização de espécies florestais adaptadas às condições locais de cada vertente pode catalisar os papéis de cada espécie na dinâmica do ecossistema, aumentando, assim, a oferta de serviços ambientais.

\section{STATUS DA SUBMISSÃO}

Recebido: 11/02/2011

Aceito: 17/11/2011

Resumo publicado online: 18/11/2011

Artigo completo publicado: 22/12/2011

AUTOR(ES) PARA CORRESPONDÊNCIA

\section{Erika Cortines}

Departamento de Ciências Ambientais, Universidade Federal Rural do Rio de Janeiro UFRRJ, Rod. BR 465, Km 7, CEP 23890-000, Seropédica, RJ, Brasil

e-mail: ecortines@gmail.com

\section{REFERÊNCIAS}

Ab'Saber A. A Serra do Japi, sua origem geomorfológica e a teoria dos refúgios. In: Morellato LP, organizador. História Natural da Serra do Japi: ecologia e preservação 
de uma área florestal do Sudeste do Brasil. Campinas: Ed. Unicamp, FAPESP, 1992.p. 12-23.

Arens NC. Variation in performance of the tree fern Cyatheacaracasana (Cyatheaceae) across a successionalmosaic in anAndeanCloud Forest. American Journal ofBotany 2001; 88(3):545-551. http:// dx.doi.org/10.2307/2657118

Arens NC, Sa' Nchez Baracaldo P. Distribution of tree ferns (Cyatheaceae) across the successional mosaic in na Andean cloud forest, Narin o, Colombia. American Fern Journal 1998; 88:60-71. http://dx.doi. org/10.2307/1547225

Arens NC, Smith AR. Cyatheaplanadae, a remarkable new creeping tree fern from Colombia, South America. American Fern Journal 1998; 88:49-59. http://dx.doi. org/10.2307/1547224

Barbieri RL, Heiden G, editores. Árvores de São Mateus do Sul e região. Brasilia: EMBRAPA Informação Tecnológica, 2009. 356 p.

Barros FA. Efeito de borda em fragmentos de floresta Montana, Nova Friburgo-RJ [dissertação]. Niterói: Universidade Federal Fluminense; 2006.

Bohrer CBA, Barros FA. Proteção e Restauração da Área do Entorno do Parque Estadual dos Três Picos: Vegetação, Uso e Cobertura do Solo. 2006. 45p. Relatório FinalProjeto CEPF-REBRAF.

Borém RAT, Oliveira-Filho AT. Fitossociologia do Estrato Arbóreo em uma Topossequência Alterada de Mata Atlântica, no Município de Silva Jardim - RJ, Brasil. Revista Árvore 2002; 26(6):727-742.

Brasil. Observações Meteorológicas - Mod. DMA -1100, período de 1931 a 1970. Brasília; 1970.

Brasil. Ministério do Meio Ambiente. Lista de espécies da flora brasileira ameaçadas de extinção. Brasília: MMA; 2008.

Brokaw, N.; Thompson, J. The H for DBH. Forest Ecology and Management v.129:89-91. 2000.

Bruijnzeel LA. Hydrology of tropical montane cloud forests: A Reassessment. Land use and Water Resources Research 2001;1:1-18.

Caiafa AN, Martins FR. Forms of rarity of tree species in the southern Brazilian Atlantic rainforest. Biodiversity and Conservation 2010; 19:2597-2618. http://dx.doi. org/10.1007/s10531-010-9861-6

Cottam, G.; Curtis, J. T. The use of distance measures in phytosociological sampling. Ecology, 37(3): 451-460, 1956.

Dawson TE. Fog in the California red Wood forest: ecosystem inputs and use byplants. Oecologia 1998;117:476-485. http://dx.doi.org/10.1007/ s004420050683
Empresa de Assistência Técnica e Extensão Rural EMATER. Levantamento da microbacia de São Lourenço: Nova Friburgo, RJ.Rio de Janeiro: EMATER; 1994. Mimeografado.

Hamilton LS, Juvik JO, Scatena FN. The Puerto Rico tropical cloud Forest symposium: introduction and workshop synthesis. In: Hamilton LS, Juvik JO, Scatena FN, editors. Tropical montane cloud forests. New York: Springer Verlag; 1995.p. 1-23.

Instituto Estadual do Ambiente - INEA. Plano de Manejo do Parque Estadual dos Três Picos. $1^{\text {a }}$ revisão. 2009. 655.p. il.

Kurtz BC, Sá CFC, Silva DO. Fitossociologia do componente arbustivo-arbóreo de florestas semidecíduas costeiras da região de Emerenças, Área de Proteção Ambiental do Pau Brasil, Armação dos Búzios, Rio de Janeiro, Brasil. Rodriguésia 2009; 60(1):129-146.

Lima HC, Guedes-Bruni RR. Diversidade de plantas vasculares na Reserva Ecológica de Macaé de Cima. In: Lima HC, Guedes-Bruni RR, editors. Serra de Macaé de Cima: Diversidade florística e conservação em Mata Atlântica. Rio de Janeiro: Instituto de Pesquisas, Jardim Botânico do Rio de Janeiro; 1997. p.29-39.

Lorenzi H. Árvores Brasileiras: manual de identificação e cultivo de plantas arbóreas do Brasil. 4rd ed. Nova Odessa: Instituto Plantarum. 1998. v. 2, 352 p.

Lorenzi H. Árvores Brasileiras: manual de identificação e cultivo de plantas arbóreas do Brasil. 4rd ed. Nova Odessa: Instituto Plantarum. 2002. v. 1, 368 p.

Lorenzi H. Árvores Brasileiras: manual de identificação e cultivo de plantas arbóreas do Brasil. 4rd ed. Nova Odessa: Instituto Plantarum. 2009. v. 3, 384 p.

Marcondes AC. Ecologia. São Paulo: Atual Editora;1996. 210p.

Marques O, Tienne L, Cortines E,Valcarce IR. Atributos ambientais definidores de presença de fragmentos florestais de Mata Atlântica em microbacias instáveis. EDUR - Revista Universidade Rural Série Ciências da Vida 2004; 24(2):145-150.

Mendonça Filho CV,Tozzi AMGA, Martins ERF. Revisão taxonômica de Machaerium sect. Oblonga (benth.) Taub. (Leguminosae, Papilionoideae, Dalbergieae). Rodriguésia 2007; 58(2):283-312.

Miranda ELF, Oliveira RR. Orquídeas rupícolas do Morro do Pão de Açúcar, Rio de Janeiro. Atas Soc. Bot. Brasil, Seção RJ 1983; 1(18):99-105.

O `Brien EM. Climatic gradients in Woody plant (tree and shrub) diversity: water-energy dynamics, residual variation, and topography. Oikos $2000 ; 89: 3$.

Oliveira RR, Zaú AS, Lima DF, Silva MBR, Vianna MC, Sodré DO, Sampaio PD. Significado ecológico da orientação de encostas no maciço da Tijuca, Rio de 
Janeiro. Oecologia Brasiliensis 1995; 1:523-541. http:// dx.doi.org/10.4257/oeco.1995.0101.28

Pacto pela Restauração da Mata Atlântica. Diversidade. [cited 2010 dez. 20].Available from: http://www. pactomataatlantica.org.br/diversidade.aspx?lang=pt-br.

Peixoto GL, Martins SV, Silva AF, Silva E. Composição florística do componente arbóreo de um trecho de Floresta Atlântica na Área de Proteção Ambiental da Serra da Capoeira Grande, Rio de Janeiro, RJ, Brasil. Acta Botanica Brasilica. 2004; 18(1):151-160. http:// dx.doi.org/10.1590/S0102-33062004000100013

Pereira JAA, Oliveira-Filho AT, Lemos-Filho J.P. Environmental heterogeneity and disturbance by humans control much of the tree species diversity of Atlantic montane Forest fragments in SE Brazil. Biodiversity and Conservation 2006; 16(6):1761-1784. http://dx.doi.org/10.1007/s10531-006-9063-4

Rolim SG, Do Couto HTZ, Jesus RM. Mortalidade e recrutamento de árvores na Floresta Atlântica em Linhares (ES). Scientia Forestalis 1999; (55):49-69.

Sá CFC. Estrutura, diversidade e conservação de angiospermas no Centro de Diversidade de Cabo Frio, Rio de Janeiro [tese]. Rio de Janeiro: Universidade Federal do Rio de Janeiro;2006.

Sant’anna Neto JL. Decálogo de climatologia do Sudeste Brasileiro. Revista Brasileira de Climatologia 2005; 1: 43 60.

Santos IS, Peixoto AL. Taxonomia do gênero Macropeplus Perkins (Monimiaceae, Monimioideae). Rodriguésia 2001;52(81):65-105.

São Paulo (Estado). Secretaria do Meio Ambiente. Fundação Florestal. Projeto Palmito Juçara: Alternativas para o manejo sustentável do Palmito Juçara. São Paulo: Imprensa Oficial. $22 \mathrm{p}$.

Satterlund DR. Wildl and Watershed Management. Ed. John Wiley\& Sons;1972. 370 p.
Seabra, R. 2009. Parque Estadual dos Três Picos é ampliado. Disponível em <http//:www.friweb.com.br/ noticias/noticia2093parque+estadual+dos+três+picos+ é+ampliado.html> acessado em: 19/09/2009.

Silva SP. Frutas no Brasil. São Paulo: Nobel; 2001.

Souza SCPM. Estratégia de regeneração de espécies arbóreas de um trecho de floresta ombrófila densa submontana no Parque Estadual de Carlos Botelho, Brasil [tese].Campinas: Universidade Estadual de Campinas; 2008

Souza MC; Morim MP. Subtribos Eugeniinae O. Berg e Myrtinae O. Berg (Myrtaceae) na Restinga da Marambaia, RJ, Brasil1. Acta Botanica Brasilica 2008; 22(3):652-683

Spolidoro MLCV. Fatores ambientais que afetam a distribuição e freqüência de capinzais na Serra do Madureira -Mendanha, Rio de Janeiro[monografia]. Seropédica: Universidade Federal Rural do Rio de Janeiro; 1998

Stehmann JR, Forzza RC, Salino A, Sobral M; Costa DPE, Kamino LHY, ediotrs. Plantas da Floresta Atlântica. Rio de Janeiro: Jardim Botânico do Rio de Janeiro; 2009. 516 p.

Sylvestre, LS; Rosa, MMT. (org.) Manual metodológico para estudos botânicos na Mata Atlântica. Seropédica, RJ: EDUR, 2002. 123 p. il.

Velloso HP, Ragel Filho ALR, Lima JCA. Manual de Classificação da vegetação brasileira adaptada a um sistema universal. Rio de Janeiro: IBGE; 1991. 124p.

Webb EL, Stanfield BJ, Jensen ML. Effects of topography on rainforest tree community structure and diversity in American Samoa, and implications for frugivore and nectarivore populations. Journal of Biogeography 1999; 26:887-897. http://dx.doi. org/10.1046/j.1365-2699.1999.00326.x 